\title{
For Your Eyes Only: Controlling 3D Online Games by Eye-Gaze
}

\author{
Howell Istance ${ }^{1,2}$, Aulikki Hyrskykari ${ }^{2}$, \\ Stephen Vickers ${ }^{1}$, and Thiago Chaves ${ }^{2}$ \\ ${ }^{1}$ Human-Computer Interaction Research Group, De Montfort University, \\ The Gateway, Leicester, LE1 9BH, UK \\ $\{$ hoi, svickers\} admu.ac.uk \\ ${ }^{2}$ Human-Computer Interaction Unit (TAUCHI), Department of Computer Sciences, \\ FIN-33014 University of Tampere, Finland \\ ah@cs.uta.fi, thiago.chaves@uta.fi
}

\begin{abstract}
Massively multiplayer online role-playing games, such as World of Warcraft, have become the most widespread 3D graphical environments with millions of active subscribers worldwide. People with severe motor impairments should be able to take part in these games without the extent of their disability being apparent to others online. Eye gaze is a high bandwidth modality that can support this. We have developed a software device that uses gaze input in different modes for emulating mouse and keyboard events appropriate for interacting with on-line games. We report an evaluation study that investigated gaze-based interaction with World of Warcraft using the device. We have found that it is feasible to carry out tasks representative of game play at a beginners skill level using gaze alone. The results from the locomotion task part of the study show similar performance for gaze-based interaction compared with a keyboard and mouse. We discuss the usability issues that arose when completing three types of tasks in the game and the implications of these for playing of this type of game using gaze as the only input modality.
\end{abstract}

Keywords: Gaze interfaces, games, evaluation, virtual communities, MMOGs.

\section{Introduction}

The popularity of Massively Multi-player Online Games (MMOGs) has increased enormously in recent years. World of Warcraft, probably the most popular fantasy role playing game, has 11 million monthly subscribers [1]. This has been accompanied by a similarly massive increase on the graphics capabilities of home machines that run the clients for these games and online worlds.

People with severe motor disabilities can derive much enjoyment from playing these games and taking part in virtual communities. Participation can be challenging and fun, it gives opportunities for social interaction, and the extent of the player's disability need not be apparent to other players. For some groups of people, eye gaze offers the only input modality with the potential for sufficiently high bandwidth to support the range of time-critical interaction tasks required to play. 
There has been much work on eye gaze interaction with 2D desktop interfaces $[2,3]$; there has been only a little work on eye gaze interaction with virtual environments, and even less work on real-time interaction with multiplayer graphical worlds. There has been some work on how eye gaze can be integrated with other input modalities for games playing by able-bodied users, but no work to our knowledge on how far gaze can be used as the only input modality to play MMOGs. Understanding how gaze can be used as a single modality for motor impaired users will have a positive carry-over for understanding how to use it as an additional modality for able-bodied users too.

A number of general problems exist with using gaze-based interaction techniques developed for 2D desktop applications for the control of 3D worlds [4]. In addition, the player is under time pressure, which poses additional demands on gaze interaction techniques. Our aim is to design a software device that will enable game playing for the users who are not able to use traditional keyboard/mouse/gamepad input devices.

In this paper we report an evaluation study of the gaze interaction techniques developed so far carried out with World of Warcraft using able-bodied participants. We present a comparison of performance data from user trials with gaze and with keyboard/mouse as the input modalities. We also discuss the main usability issues associated with the gaze condition that arose during the game playing tasks.

\section{Related Work}

Interest in using gaze-input in games has been increasing due to the naturalness of pointing, and the potential for additional attentive input that the user's gaze can provide.

Isokoski et al. [5,6] used a first person shooter (FPS) style game in order to assess the performance of eye gaze as an extra input modality to mouse and keyboard. Their first findings showed that using eye gaze for aiming will not always improve the performance of the players when compared to using the game controller for aiming. However they did find that the number of hits from gaze is comparable to using the game controller alone, and that using gaze to play was more entertaining. The possibility of using eye gaze for controlling player direction was briefly examined but due to the necessity of the user constantly needing to change direction it was deemed not feasible.

Smith and Graham [7] performed an experiment using a similar control system on an open source port of the FPS Quake 2 called Jake2. Similar to Isokoski, the authors did not find any advantage in performance with gaze. However, their subjective user results showed that using eye gaze offered a much more immersive experience than using a mouse and keyboard.

Increased levels of immersion and enjoyment were also found by Jönsson [8] during trials using a combination of eye gaze and mouse within the FPS Half Life. Smith and Graham also performed trials using a version of the 80's arcade game Missile Command. Participants were required to use eye gaze to target missiles that were falling from the top of the screen and press a button to shoot them. They found that there is a need to fire ahead of the missile for a successful hit and this is easily achievable using a mouse. However, it is extremely difficult when using eye gaze to 
fire ahead as there is a constant distraction of the missile itself (the users looked at the missile rather than where they wished the missile to go). Thus, the majority of eye gaze shots missed and fell behind the missile, demonstrating the importance to disambiguate between a users attention and their intention when implementing interaction techniques.

Various implementations of different gaze driven paddle games (e.g. $[9,10])$ where simply following the ball by gaze gives the paddle the optimal coordinates show how effective gaze can be when used in a natural way. This point was made long ago by Jacob who advocated using gaze for non-command-based interaction, rather than deliberate command-based interaction [11]. Good task candidates are the ones where the user has to make a move to a point of interest (bat to ball in this case), or perhaps in World of Warcraft, move from 'here' to a target object, such as an enemy character, by simply looking at that target object.

Recently, Isokoski et al. [12] has reviewed the potential of using eye gaze in different genres of gaming as an additional modality for able bodied gamers. They identify features of each genre that are favourable or unfavourable for gaze control. They raise the important point that modifying a game to facilitate gaze control may remove some of the challenges and requisite skills that make playing the game interesting.

In context of immersive virtual environments (rather than games) Tanriverdi and Jacob [13] investigated gaze-based interaction techniques for selecting objects and compared performance using gaze with using a handheld pointer. Objects were assigned an index of interest determined by how long and often the user looked at them and were automatically selected and zoomed in upon. Significant performance benefits were found particularly for objects distant from the user in virtual space. They also found there was a cost in terms of poorer spatial memory of the locations of objects in the world in the gaze conditions. This is of particular interest for gaze-based interaction with games, when a significant amount of a user's visual attention may be allocated to interacting with the game, rather than observing the environment.

\section{Design of the Eye-Gaze Based Games Interaction Device}

Our overall objective is to produce a software device that uses eye position and gaze patterns as input, and produces keystroke and mouse events as output. The game client reacts to these events as if they had come from the keyboard and mouse hardware devices. In this way the device can be used with any game that can be operated by a keyboard and mouse. When the user gets a new game or joins a new on-line community, it should be easy to configure the eye device for the new game. Consequently the device should not require any modifications to the game client software.

Pointing using gaze measurement is inherently inaccurate. The eye is being used for interacting with on-screen objects as well as looking at the game. Normal keyboard and mouse use utilizes both hands for very precise rapid movements in parallel with the use of the eyes. We have no expectation that gaze interaction will be as good as keyboard and mouse for all aspects of games playing and all skill levels of players. However we do want to understand which parts of playing a particular genre of game, and at which skill level of play, gaze based interaction comes close to 
conventional input devices. For these tasks and at this skill level at least, the player supported by gaze need not appear to be different when on-line from their able-bodied counterparts.

\subsection{Principles of the Design Solution}

Our device [4] maps patterns of gaze behavior into various keyboard and mouse events. Each mapping corresponds to a mode which defines how the user's gaze behavior is interpreted. We can select four modes at any one time, which are then assigned to the four edges of the screen (see Fig. 1). The user can switch between modes by glancing off a particular edge of the screen and back again. Feedback about the currently active mode is given by a green strip that appears along the edge of the screen indicating the active mode. Additional feedback is given by changing the system cursor but this is unreliable as some games will define their own cursors.

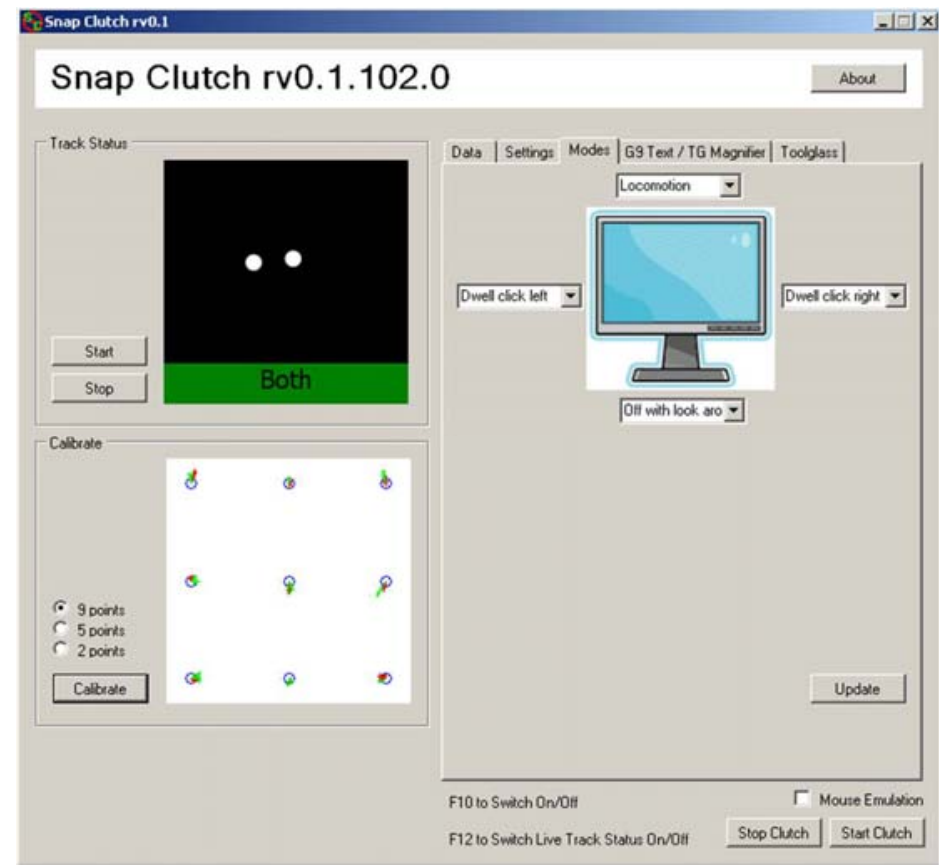

Fig. 1. The configuration window of the device in which the user defines the mapping of the desired modes

\subsection{Gaze Interaction Performance Estimates}

We obtained performance data for gaze interaction with a previous version of the device from an evaluation study using Second Life [14]. Twelve participants were required to do set of three tasks with keyboard and mouse and a similar set of three tasks with gaze. The tasks were designed to represent locomotion, object manipulation and application control. 
The results were encouraging and showed that all participants were able to complete all tasks, after only a brief introduction and training with Second Life and with gaze interaction. Task times between gaze and keyboard/mouse were compared for each task, and these were partitioned into 'error time' and 'non-error' time. The proportions of each type of error enabled predictions to be made about the performance benefits that could be expected if the respective causes of each type of error were to be designed out. The main error types found were locomotion errors and accuracy errors. The first type resulted from a lack of sufficient control over the avatar's direction and speed of movement in the virtual world. The second type resulted from difficulty in positioning the cursor over small targets in interface control objects long enough for the dwell period to expire and the click event to be generated. The latter problem is common with gaze-based interaction. Table 1 shows the ratios of the task times with and without the error time component. In the present study we wished to see whether the non-error time performance ratios were achievable following modifications to our software device when using World of Warcraft (as an example of a popular MMORPG).

Table 1. Ratio of task time components from first evaluation study

\begin{tabular}{lcc}
\hline Second Life Task & $\begin{array}{c}\text { Total task times } \\
\text { KB/Mouse : Gaze }\end{array}$ & $\begin{array}{c}\text { Non-error time } \\
\text { KB/Mouse : } \\
\text { Gaze }\end{array}$ \\
\hline Locomotion & $1: 1.6$ & $1: 1.2$ \\
In-World Object & $1: 4.6$ & $1: 2.0$ \\
$\begin{array}{l}\text { Manipulation } \\
\text { Application Control }\end{array}$ & $1: 2.8$ & $1: 2.5$ \\
\hline
\end{tabular}

\subsection{The Present Design of the Gaze Interaction Device}

The locomotion mode uses 'active regions' of the screen. When the user is in this mode, different keystroke events - which control locomotion - are automatically generated and sent to the game client application. Many games use the convention that the ' $w$ ' key moves the character forward, the ' $a$ ' key to the left, the ' $d$ ' key to the right, and the 's' moves the character backwards. The cursor control keys usually have the same function. We found that a player's eye movements using a mouse and keyboard in World of Warcraft, stayed in quite a distinctive area in front of the avatar. In Fig. 2 there is a heat map visualization [15] of a player's gaze positions during a period of movement around in an unfamiliar part of a world. On the basis of this we defined regions of the screen that the user does not usually look into during normal navigation. These are also shown overlaid on Fig. 2, although these are not visible to the player during use.

When the user looks at the regions, 'w', 'wa', 'a', 's', 'd', and 'wd' keystrokes respectively are streamed to the games client application. The first evaluation in Second Life showed that turning using gaze was very sensitive and often caused overshooting that required a steering correction in the opposite direction. To smoothen 


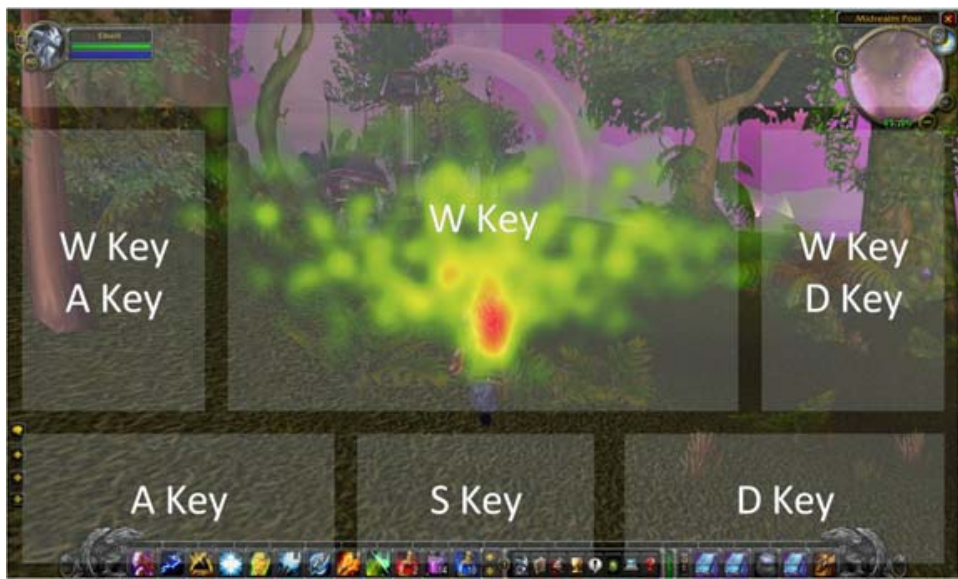

Fig. 2. Heat map illustrating the gaze behavior of a player when moving around in a part of the world in World of Warcraft that he was unfamiliar with

the turn, regions on the right and left send the ' $w$ ' key interleaved with the turning ' $a$ ' and 'd' keys. Looking down and left (/ right) still sent just a ( / d) keys to the application, and seemed to match surprisingly well the participants' intuitive expectations. To stop locomotion, the participant glanced down to switch into 'no action' mode.

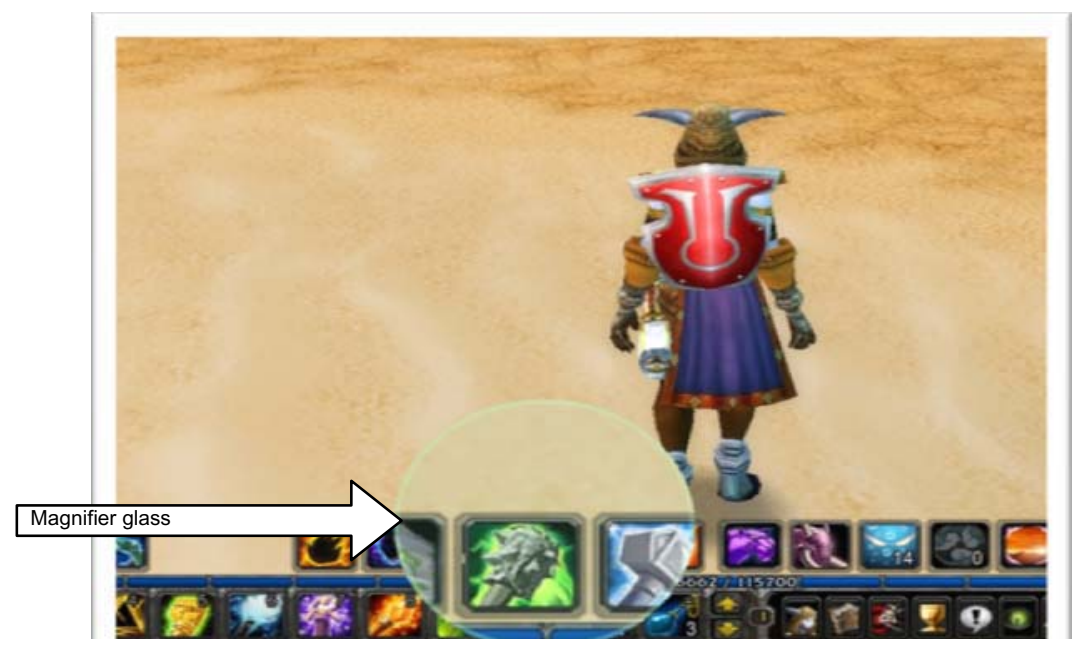

Fig. 3. The magnifier glass can be dropped by dwell to a location where a close-up manipulation is needed 
The magnifier glass (see Fig. 3) was designed to counteract the accuracy problem. The user can pick up the magnifier glass by a dwell on a semi-transparent icon placed on the game window. The magnifier glass then follows the point of gaze until the user drops it with another dwell. When the magnifier glass is dropped the user can then dwell within the magnifier area and send other interaction events within the magnifier glass area. A dwell outside the magnifier area moves the glass to a new position, and a subsequent dwell on the magnifier icon turns the magnifier off. The transparency, location and size of the magnifier icon can be configured from the device settings (Fig. 2) to reduce its interference with the underlying screen.

\section{Testing the Modified Device with World of Warcraft}

We carried out a series of user trials with the modified gaze interaction device to study its usability when playing World of Warcraft. Unlike the first study in Second Life, this study included time-constrained interaction with other characters. We also wanted to see whether we could obtain similar performance ratios of keyboard (and mouse) to gaze to those expected from the first study. World of Warcraft is an MMORPG in which the player's character or avatar plays alone or with other players to complete quests. The play involves fights with monsters or other players. These fights involve the use of hand-held weapons or spells which can be cast on opponents. The player has a collection of equipment which can be worn or sold and which can be taken, or 'looted', from opponents when they have been defeated in a fight. A player can have increasing levels of experience as a result of acquiring skills and using them to defeat opponents. In this study, we were only interested in tasks representative of beginners' level experience. The rationale here was that if these are achievable by gaze only, then we can progressively increase the difficulty of the tasks to establish the limits of what is possible using gaze interaction only. A character was created with a medium experience level (level 16) and all trials were carried with this character in the same virtual space around a village. We used a public server so there were other characters in the same space. We wanted the tasks to be conducted in a realistic play environment with a reasonable level of random distraction caused by external events in the game.

\subsection{Device Configuration}

For the user trials we used the following modes. (1) Glance Up: 'Locomotion' mode, which functions as described in Section 3.3. (2) Glance Down: 'No action with look around' mode, in which gaze dwell invokes no action, but the character rotates when the user looks inside the left and right hand edges of the screen. (3) Glance Left: 'Left mouse button click' mode, in which a dwell causes a left button click event. (4) Glance Right: 'Right mouse button click' mode, in which a dwell causes a right button click event. (5) Glance at Magnifier icon: 'Magnifier' mode, whose operation was described in Section 3.3. 


\subsection{User Trials}

Tasks. We designed four tasks representative of beginner level play in the game. The tasks were chosen following a task analysis of a period of beginners play in an MMORPG. These were:

- Locomotion task to walk to a location identified on the inset map, to turn around and return the starting point; and then to repeat the task running. There was no control over character speed and the participant was asked to stay on the path and complete the task as quickly as possible.

- Fighting task to find and fight a level 3 monster. The participant was asked to cast the same spell as many times as possible during the fight (by left clicking on an icon located on the shelf in the centre bottom of the screen). The difference in levels assured the participant would always win. After the fight, the participant was asked to loot the corpse (by right clicking on it) of one item (by left clicking on the list of treasure).

- Equipment task to put on or wear four items of equipment by opening a pouch (left clicking its icon in the bottom right of the screen); then opening the character sheet (left clicking its icon also in the bottom right of the screen); then selecting an item from the pouch (left clicking on its icon in the pouch); then selecting the highlighted slot in the character sheet which was open in the upper left part of the screen (again by left clicking in the empty slot); then closing both windows (left click in the close box in the top right of the window)

- Communication task to greet an object by typing a sentence using a predictive text keypad and then respond to the objects reply by typing another sentence and a closing abbreviated remark. We had designed and implemented the keypad to support communication with other players. There were a number of problems that arose during the trials with this part of the device and the outcomes of this task are not presented in this paper.

Participants. Ten participants were recruited for the trials, aged between 18 and 44 . These were 9 males and 1 female, all were able-bodied, and all were students or staff at the computer science department at the university (biased gender distribution is justified on the basis that gamers are mostly males). None had taken part in the first experiment. Five had current extensive games playing experience with MMORPGs, three with World of Warcraft. All of the other 5 had played computer games, but did not consider themselves to be experienced MMORPG players. Participants were given cinema tickets in return for taking part.

Procedure. We carried out the trials in a usability laboratory equipped so that the trials could be observed from an adjacent room, separated by a one-way glass window. A Tobii T60 was used for the trials. The screen image from the trial machine was visible in the viewing room and it was recorded for subsequent video analysis.

Each trial consisted of a training phase (50 to 60 minutes), a break (20 to 30 minutes), and the data collection phase (about 30 minutes). The first part of the training covered the use of the gaze device, the magnifier, and locomotion mode. The second part of the training consisted of a structured introduction to World of Warcraft 
and completing a set of standard tasks. This was done first by keyboard and mouse, and then with gaze. After a break, all the four tasks were recorded with the keyboard and mouse. The same four tasks were then carried out using gaze. After the fourth task there was a 10 minute interview. The order of conditions during the trials was not counterbalanced as we wanted to increase the practice obtained before the gaze trial. We had no expectation that gaze would perform better than keyboard and mouse.

\section{Results}

\subsection{Locomotion Task}

In the present study, the locomotion task was carried out both running and walking. All participants completed the task in both conditions. Table 2 shows the means and standard deviations of the task completion times for the 9 participants. Data from one participant was omitted from the quantitative analysis but retained in the analysis of subjective data. This was due to problems calibrating the eyetracker. Willcoxen's Matched Pairs Signed Ranks Test shows the difference between the two conditions not to be significant $(\mathrm{p}(\alpha)>0.05)$ when participants were walking. Running however took significantly longer in the gaze condition $((\mathrm{p}(\alpha) \approx 0.01)$ compared with the keyboard and mouse condition.

Table 2. Locomotion total task times for $\mathrm{Kb} / \mathrm{M}$ and gaze

\begin{tabular}{crccc}
\hline \multicolumn{2}{l}{ Locomotion } & Kb/M (s) & Gaze (s) & Kb/M:gaze (ratio) \\
\hline \multirow{4}{*}{ walk } & mean & 80.9 & 83.2 & $1: 1.0$ \\
& stdev & 2.6 & 7.5 & \\
& $\mathrm{n}$ & 9 & 9 & $1: 1.1$ \\
\multirow{2}{*}{ run } & mean & 29.6 & 32.7 & \\
& stdev & 1.4 & 2.5 & \\
& $\mathrm{n}$ & 9 & 9 &
\end{tabular}

In both cases the keyboard and mouse to gaze performance ratio was better than expected from the locomotion task in the Second Life trials (Table 1).

In the subjective evaluation, 7 participants of the 10 participants said controlling the rate of turn of the character was especially difficult in the gaze condition. Fine control of changes in direction was said by some participants to be much easier with the keyboard than with gaze. The other control issue reported by 3 was the difficulty in starting and stopping movement quickly in the gaze condition (by glancing over the bottom edge of the screen). Also searching for a type of monster required reading the labels over the heads of characters as they appeared on screen. If these appeared on the right or left sides, reading the labels would cause unintentional turns in that direction. Another participant referred to the problem of feedback where it was difficult to see whether the characters had turned far enough when looking at the bottom left or right hand corners of the screen. Three participants rated gaze control of locomotion to be easier than keyboard and mouse as there was no need to keep pressing a key to move. 
We tried to rectify the 'turn overshoot' problem identified in the Second Life evaluation study by interleaving forward and sideways key events during a turn. However we still observed many instances of this error, particularly in the gaze condition. These did not result in significant recovery time loss but they did lead to more deviations from the centre of a forward path movement. Another observed gaze specific error was a 'distraction' error, where another character took the participant's visual attention to part of the screen which caused the own character to turn. This also caused path deviation, which had to be corrected.

\subsection{Fighting Task}

All participants completed the task in both conditions. The data from the fighting task is shown in Table 3. This shows the duration of the fight and the numbers of spells cast during the fight. The duration was measured from when the own character first engaged the monster until the monster died. The gaze fight lasted twice as long as the fight in the keyboard/mouse condition because the number of spells cast was fewer.

Table 3. Time taken and numbers of spells cast during the fighting task

\begin{tabular}{|c|c|c|c|c|}
\hline \multicolumn{2}{|c|}{ Fighting } & \multirow{2}{*}{$\frac{\mathbf{K b} / \mathbf{M}}{5}$} & \multirow{2}{*}{$\frac{\text { Gaze }}{3}$} & $\begin{array}{c}\text { Kb/M:gaze } \\
\text { (ratio) }\end{array}$ \\
\hline number of & median & & & \\
\hline spells cast & $\mathrm{n}$ & 9 & 9 & \\
\hline \multirow{3}{*}{ time } & mean & 15.1 & 31.69 & $1: 2.1$ \\
\hline & stdev & 2.67 & 17.82 & \\
\hline & $\mathrm{n}$ & 9 & 9 & \\
\hline
\end{tabular}

In this simplified fighting task, the main requirement was to click the spell icon continuously to cast as many spells as possible. Willcoxen's Matched Pairs Signed Ranks Test shows the difference in the tasks times between the two conditions to be significant $(\mathrm{p}(\alpha)<0.01)$

In the subjective evaluation, 5 of the 10 subjects considered the size and location of the spell buttons to be a major factor with the difficulty of the task in the gaze condition. The magnifier was not used by any of the participants. When asked whether they considered using this to select the spell, one participant said that the number of actions to get the magnifier, drop it and then select the spell was simply too distracting from the action during the fight. This is an important indicator for the design of gaze interaction techniques for this type of task which involves interaction with other characters.

Two participants said it was difficult to control the character during the fight as it was not possible to do multiple actions at the same time, such as moving and casting spells. This is a requirement for some classes of character but not for others. There is one class of character that has an agent (a pet) that can fight on its behalf, which offers one type of solution to the issue of gaze-controlled fighting. Another participant pointed to the difficulty of gaze selecting a monster to engage in a fight while it was moving as the location for the dwell event has to be anticipated before the dwell begins. Another participant noted how difficult it was not to look at the battle while they needed to keep looking at the spell button in the tool bar at the bottom of the screen. 
This task required rapid changing between modes to move, engage the character with a right click and then to cast spells with a left click. Three participants noted that they found changing modes quickly by glancing off screen difficult, although they thought the situation might improve with more practice.

\subsection{Equipment Task}

All participants completed the task in both conditions. The results are shown in Tables 4 and 5 . The task has been split into 2 parts, opening the pouch window and the character sheet window (Table 4), and moving each of the four items from the pouch to the character sheet (Table 5). The icons to open the two windows were situated at the edge of the screen and some participants found selecting these by gaze particularly difficult due to the tracking accuracy near the edge of the calibrated area.

Table 4. Number of clicks and time taken for the first part of the equipment task

\begin{tabular}{crccc}
\hline \multicolumn{2}{c}{ Opening 2 windows } & Kb/M & Gaze & Kb/M:gaze (ratio) \\
\hline number of & median & 2 & 3 & \\
clicks & $\mathrm{n}$ & 9 & 9 & \\
\hline \multirow{2}{*}{ time } & mean & 3.4 & 17.2 & $1: 5$ \\
& stdev & 1.1 & 14.6 & \\
& $\mathrm{n}$ & 9 & 9 & \\
\hline
\end{tabular}

Table 5. Number of clicks and time taken for the second part of the equipment task

\begin{tabular}{crccc}
\hline \multicolumn{2}{c}{ Moves 4 items } & Kb/M & Gaze & Kb/M:gaze (ratio) \\
\hline number of & median & 8 & 14 & \\
clicks & $\mathrm{n}$ & 9 & 9 & $1: 2.6$ \\
\multirow{2}{*}{ time } & mean & 17.4 & 45.4 & \\
& stdev & 5.4 & 23.0 & \\
& $\mathrm{n}$ & 9 & 9 & \\
\hline
\end{tabular}

The keyboard/mouse to gaze performance ratios for the first and second parts of the tasks were 1:5 and 1:2.6 respectively. This gives a measure of the difference in difficulty between the two parts. Some participants used the magnifier in the gaze condition but only after they had tried to select the targets unaided. This resulted in long times on task and the standard deviations in both of the tables above reflect the large variability in task times. Also dropping the magnifier at the bottom of the screen meant that half of the magnifier was clipped, which could, in some cases, obscure the enlarged view of the target icon.

In the subjective evaluation, opinion was divided between those who thought the task was easy to complete and those who found the first part (opening the equipment windows) and consequently the whole task difficult. 4 of the 10 participants rated the ease of the task completion with gaze as being either as easy as or easier than with mouse and keyboard. There may be an order effect as this task always followed the fighting task in both the gaze and the keyboard and mouse conditions, and may have been considered easier overall. 


\section{Discussion}

The outcomes of the trials have demonstrated the feasibility of gaze control of MMORPGs in as much that all participants were able to complete all of the tasks.

There is no universal definition of 'beginner' in terms of skills. Once a player knows what to do in the game, how to level the character, where to buy equipment and what spells do, he or she is no longer a beginner but a novice. Getting to that stage does not take very long (perhaps 30 minutes of play), but getting beyond this stage takes a much longer time. We believe that we demonstrated that gaze control of novice play is achievable.

We have used the ratio of task time using gaze to the time taken to complete the same task with keyboard and mouse as the main quantitative performance indicator. This allows some comparisons to be made between games (or worlds) provided the limits of similarities between the games and their tasks are recognized. The first experiment carried out with Second Life suggested that if the causes of identified problems in controlling locomotion could be designed out, then a performance ratio of keyboard/mouse to gaze in the region of $1: 1.2$ could be expected. We obtained performance ratios of $1: 1.1$ or better in these trials. The main problem with gaze control of locomotion is the lack of fine control over the rate of turn of the character. To some extent, this is a problem with the game client as well as with gaze, and there have been some discussions on forums about the need for better rate of turn control when using keyboard and mouse control with the World of Warcraft client. We recognise that the task given to participants was restricted to moving in a fixed path, and not moving in response to dynamic events in the game.

The fighting task shows some of the real limitations of using gaze to emulate normal mouse and keyboard without modifying the interface. The fighting task was deliberately chosen so that the participant character would always win and casting the same spell repeatedly is a very simplified view of fighting. The trials also revealed the limited nature of moded interaction in the present configuration of the interface, that is, that the player could either move, or cast spells, but not do both at the same time.

There was a 'midas-touch' like problem when the participant looked at a character that appeared at the edge of the screen when looking for monsters which also caused an unwanted change in direction.

The equipment and the fighting task were both hampered by the familiar problem of the difficulty of selecting small targets using gaze. A number of icons in the interface configuration we used were located right at the bottom of the screen, which lead to problems with the eye tracker calibration accuracy. The version of the magnifier that we developed as a means of overcoming accuracy problems apparent from the first experiment was not an effective solution to these. Some of these problems could be attributed to specific implementation issues and some to the lack of training the participants had with the interaction technique. However the main problem appeared to be the means invoking the magnifier, moving it, dropping it, and clicking through it were just too distracting and time consuming for it to be effective in a timeconstrained game playing situation. An alternative means of using the magnifier needs to be found, or an alternative solution altogether to the accuracy issue is needed. 
The equipment changing task shared some similarities with the appearance tasking changing task in Second Life. That experiment suggested that if the accuracy issues with gaze selection could be resolved then a performance ratio of $1: 2.5$ could be expected. The part of the equipment task involving object selection away from the edge of the screen in these trials had a keyboard/mouse to gaze ratio of $1: 2.6$. The similarity in these ratios gives encouragement to the idea that gaze performance across games can be quantified using the ratio as a metric, and that there is some consistency between similar types of task.

There are also broader interaction issues that the study has raised. In normal interaction in World of Warcraft, information about characters or equipment, for example, is displayed as text in a pop-up box in response to a mouse rollover. Dwell is fundamentally unsuitable as a means of rolling the mouse pointer over elements. The player will read what the box contains and in so doing will move the gaze point off the element. Alternative gaze actions for selecting elements, other than dwell, are needed.

The trials show that where we have time constrained game play, then gaze based emulation of mouse actions using dwell on standard interfaces is too limited. An interface configuration which allows the player to issue rapid commands with visual attention being diverted from the centre of the screen as little as possible is needed. Our ideas here involve using gaze based gestures, and a prototype gaze gesture driven interface to World of Warcraft has been built, and is currently being tested.

\section{Conclusions}

This work should be considered as a first step towards gaze-based game interaction for motor impaired users. We have not yet tested the interface with such users nor have we explored fully the range of design variables necessary to accommodate different types of motor impairment. We do, however, believe that the objective of total gaze control is achievable for a large proportion of users with motor impairments. The same interface works with an example of an MMORPG (World of Warcraft) and with an example of a multi-user virtual community (Second Life) and we expect it will work, with minor adjustments, with other games in each of these genres.

The difference between this project and others that have investigated eye gaze as a modality for game playing is the emphasis in this work on gaze as the sole input modality to enable motor impaired people to play MMORPGs. Others have studied how gaze can be used to complement other input modalities for use by able-bodied gamers. We have been able to demonstrate that it is feasible to carry simple locomotion, fighting and equipment manipulation tasks using gaze alone in World of Warcraft. From earlier work with gaze control of Second Life, we generated some expected performance differences between gaze and keyboard/mouse interaction using task time ratios for similar types of task. In this study we found good agreement with these expected values. The study has also highlighted the limitations of the current approach to using gaze for time-constrained interaction with World of Warcraft as an example of an MMORPG. If gaze-based interaction with MMORPGs is to be realised then interaction techniques which are lightweight, rapid and allow the user to maintain their attention on the centre of the screen are needed. This leads to alternative approaches to gaze interaction that embody these requirements, which are currently under investigation. 
Acknowledgments. This work is supported by: Communication by Gaze Interaction (COGAIN) FP6 Network of Excellence, the Institute of Creative Technologies (IOCT) at De Montfort University, the Royal Academy of Engineering London and the Academy of Finland.

\section{References}

1. WoW Insider (2008), http: / / www. wowinsider.com/2008/10/28/ world-of-warcraft-hits-11-million-subscribers-worldwide/

2. Hyrskykari, A., Majaranta, P., Räihä, K.-J.: From Gaze Control to Attentive Interfaces. In: Proc. HCII 2005, Las Vegas, NV (July 2005), http://www.cs.uta.fi/hci/gaze/docs/Hyrskykari-HCII2005.pdf

3. Majaranta, P., Räihä, K.-J.: Twenty years of eye typing: systems and design issues. In: Proc. Symposium on Eye Tracking Research \& Applications (ETRA 2002), pp. 15-22. ACM Press, New York (2002)

4. Istance, H., Bates, R., Hyrskykari, A., Vickers, S.: Snap clutch, a moded approach to solving the Midas touch problem. In: Proc. Symposium on Eye Tracking Research \& Applications (ETRA 2008), pp. 221-228. ACM, New York (2008)

5. Isokoski, P., Martin, B.: Eye tracker input in first person shooter games. In: Proc. Communication by Gaze Interaction; COGAIN Conference 2006, pp. 76-79 (2006)

6. Isokoski, P., Hyrskykari, A., Kotkaluoto, S., Martin, B.: Gamepad and eye tracker input in fps games; data for the first 50 minutes. In: Proc. Communication by Gaze Interaction; COGAIN Conference 2007, pp. 11-15 (2007)

7. Smith, J., Graham, T.C.N.: Use of eye movements for video game control. In: Proc. ACM SIGCHI international conference on Advances in computer entertainment technology (ACE 2006). ACM, New York (2006)

8. Jönsson, E.: If looks could kill - an evaluation of eye tracking in computer games, Master's thesis, Royal Institute of Technology, Stockholm (2005)

9. LC Technologies, Eyegaze software (2009), http: / / www . eyegaze.com/content/ programs-eyegaze-edge-communication-system

10. Dorr, M., Bohme, M., Martinetz, T., Barth, E.: Gaze beats mouse: a case study. In: Proc. Communication by Gaze Interaction; COGAIN Conference (2007)

11. Jacob, R.J.K.: Eye Movement-Based Human-Computer Interaction Techniques: Toward Non-Command Interfaces. In: Hartson, H.R., Hix, D. (eds.) Advances in Human-Computer Interaction, vol. 4, pp. 151-190. Ablex Publishing Co., Norwood (1993)

12. Isokoski, P., Joos, M., Spakov, O., Martin, B.: Gaze Controlled Games, to appear in UAIS. Journal of Universal Access in the Information Society (2009)

13. Tanriverdi, V., Jacob, R.: Interacting with eye movements in virtual environments. In: Proc. SIGCHI Conference on Human Factors in Computing Systems (CHI 2000). ACM Press, New York (2000)

14. Istance, H., Hyrskykari, A., Vickers, S., Ali, N.: User Performance of Gaze-based Interaction with On-line Virtual Communities. In: Proc. COGAIN Conference 2008, pp. 28-32 (2008)

15. Wooding, D.: Fixation maps: Quantifying eye-movement traces. In: Proceedings of the Symposium on Eye Tracking Research \& Applications, pp. 31-36. ACM Press, New York (2002) 